\title{
MULTIPLICITY OF SINGULAR VALUES FOR TENSORS*
}

\author{
K.C. CHANG $^{\dagger}$ AND TAN ZHANG $\ddagger$
}

\begin{abstract}
In linear algebra, both eigenvalue problems and singular value problems for matrices are of fundamental importance. In this paper, we use critical point theory from nonlinear analysis and tools from algebraic topology to study the existence and multiplicity problem of singular values in the higher order tensor setting.
\end{abstract}

Key words. Singular value, eigenvalue, tensor, Liusternik-Schnirelmann theory, category, cuplength.

AMS subject classifications. $\quad$ 15A18, 15A69, 55M30, 58C40.

\section{Introduction}

Higher order tensors are generalizations of matrices; a matrix is a second order tensor. Tensors have numerous applications in many branches of mathematics and physics. In addition to the classical areas, such as differential geometry, elasticity, and gravitation theory, they have been involved in various new areas of applied mathematics, e.g., higher order statistics (HOS), signal processing, medical image processing; see for instance [12]. Many important ideas, notions, and results have been successfully extended from matrices to higher order tensors. Among these, in particular, are the notions and certain basic algebraic and geometric properties of rank, eigenvalue/eigenvector, and singular values, for higher order tensors, see [8, 14, 12, 18]. Decomposition techniques and algorithms for tensors are also developed in these settings [13].

The concept of eigenvalues for tensors was first introduced and studied by Qi [18] and $\operatorname{Lim}$ [14]. Qi proved the existence of a real eigenvalue for a symmetric tensor, and Lim proved the existence of a nonnegative eigenvalue for an irreducible tensor with nonnegative entries. Moreover, Qi introduced the characteristic polynomial for a symmetric tensor via multi-dimensional determinants of polynomial systems, by which he was able to count the number of complex eigenvalues of a symmetric tensor. As a parallel subject, the notion of singular values for a tensor was introduced in [12] (see also [14]); it is used in the canonical decomposition of a tensor. The existence of a singular value was also proved in [14] via a variational approach.

Unfortunately, due to the nonlinear nature of the eigenvalue and the singular value problem for tensors, methods in classical linear algebra and matrix theory cannot be applied directly. Although methods of algebraic geometry are powerful and effective over the complex field, in dealing with the real eigenvalue/singular value and the real eigenvectors, we appeal to topological methods. Previously, we have extended the result of the existence of $n$ eigenvectors for a symmetric $n \times n$ matrix to tensors by the classical Liusternik-Schnirelmann theory [4], and we have also extended the classical Perron-Frobenius theorem for irreducible nonnegative matrices to irreducible nonnegative tensors by the Brouwer fixed point theorem [3].

The main purpose of this paper is to generalize the well known result on singular

\footnotetext{
${ }^{*}$ Received: April 3, 2009; accepted (in revised version): April 20, 2009. Communicated by Shi Jin.

${ }^{\dagger}$ School of Math. Sci., Peking Univ., Beijing 100871, China (kcchang@math.pku.edu.cn).

${ }^{\ddagger}$ Dept. Mathematics \& Statistics, Murray State University, Murray, KY 42071, USA (tan.zhang@ murraystate.edu).
} 
values of a matrix to the higher order tensor setting, i.e., there exist $r$ pairs of positive/ negative singular values $\pm c_{1}, \cdots, \pm c_{r}$ for an $n \times m$ rectangle matrix with rank $r$. More sophisticated tools from algebraic topology are required.

For a $(p, q)$ order $(n, m)$ dimensional real tensor $\mathcal{A}=\left(a_{i_{1} \cdots i_{p} j_{i} \cdots j_{q}}\right)$, a real number $\lambda$ is called a singular value of $\mathcal{A}[5]$ if there exists $(x, y) \in\left(\mathbb{R}^{n} \backslash\{0\}\right) \times\left(\mathbb{R}^{m} \backslash\{0\}\right)$, satisfying the system:

$$
\left\{\begin{array}{l}
\sum_{1 \leq i_{2}, \cdots, i_{p} \leq n, 1 \leq j_{1}, \cdots, j_{q} \leq m} a_{i, i_{2}, \cdots, i_{p}, j_{1}, \cdots, j_{q}} x_{i_{2}} \cdots x_{i_{p}} y_{j_{1}} \cdots y_{j_{q}}=\lambda x_{i}^{p+q-1}, 1 \leq i \leq n \\
\sum_{1 \leq i_{1}, \cdots, i_{p} \leq n, 1 \leq j_{2}, \cdots, j_{q} \leq m} a_{i_{1}, \cdots, i_{p}, j, j_{2}, \cdots, j_{q}} x_{i_{1}} \cdots x_{i_{p}} y_{j_{2}} \cdots y_{j_{q}}=\lambda y_{j}^{p+q-1}, 1 \leq j \leq m .
\end{array}\right.
$$

As before, if $p+q$ is even, we can reformulate the problem as a variational problem, and then apply the critical point theory to the function:

$$
f_{\mathcal{A}}(x, y)=\sum_{1 \leq i_{1}, \cdots, i_{p} \leq n, 1 \leq j_{1}, \cdots, j_{q} \leq m} a_{i_{1}, \cdots, i_{p}, j_{1}, \cdots, j_{q}} x_{i_{1}} \cdots x_{i_{p}} y_{j_{1}} \cdots y_{j_{q}},
$$

on the product of spheres $S^{n-1} \times S^{m-1}$, when $n, m \geq 2$.

Our approach is divided into two parts: $p, q$ are both even or $p, q$ are both odd. We study them separately. Here is a brief summary of our main results of this paper: When $p, q$ are both even, since the function $f_{\mathcal{A}}(x, y)$ is even in both variables $x$ and $y$, the problem is reduced to a function on the product space of two real projective spaces $\mathbb{R} \mathbb{P}^{n-1} \times \mathbb{R} \mathbb{P}^{m-1}$. Consequently, we focus on the estimation of the L-S category and the cup-length of the space.

TheOREM 1.1. If $p$ and $q$ are both even, then, counting multiplicities, $\mathcal{A}$ possesses at least $m+n-1$ singular values.

When $p, q$ are both odd, the function $f(x, y)$ is invariant under the transformation $(x, y) \mapsto(-x,-y)$, which generates a two element group $\mathcal{G}$ isomorphic to $\mathbb{Z}_{2}$. We investigate the mod-2 cohomology ring of the space $S^{n-1} \times S^{m-1} / \mathcal{G}$ to estimate its L-S category cat $_{\mathcal{G}}$. We obtain the following:

TheOREM 1.2. If $p$ and $q$ are both odd, then, counting multiplicities, $\mathcal{A}$ possesses at least cat $_{\mathcal{G}} \geq \min (n, m)+1$ singular values.

As a byproduct, a Borsuk-Ulam type theorem is formulated. Although the lower bound for the number of singular values may not be sharp in general, in a few cases, the results can be improved.

It is worth pointing out that when $p$ and $q$ are both odd, tensors are similar to matrices in the sense that singular values appear in pairs. We shall explore these properties in the last section of the paper.

By introducing the Condition (S) (see section 4), a more in-depth investigation on the topology of the level sets for the function $f_{\mathcal{A}}$ is conducted. Let $\Lambda$ be the eigen-set corresponding to the singular value 0 ; we prove the following:

Theorem 1.3. Assume that $\operatorname{cat}_{\mathcal{G}}(\Lambda)=s$, and that Condition (S) holds. If $p$ and $q$ are both odd, then, counting multiplicities, the function $f_{\mathcal{A}}$ has at least $r-s$ positive and $r-s$ negative critical values; in particular, counting multiplicities, $\mathcal{A}$ has at least $r-s$ positive and $r-s$ negative singular values, where $r=\min \{n, m\}$.

This theorem yields the result on singular values for matrices as a special case. 
Our paper is organized as follows: in section 2, we introduce the basic notions and results from $[4,5]$, and [18]. The lifting tensor $\mathcal{T}_{\mathcal{A}}$ of a rectangle tensor $\mathcal{A}$ is defined, and then we establish the relationship between the eigenvalues for $\mathcal{T}_{\mathcal{A}}$ and the singular values for $\mathcal{A}$. Moreover, we study the special case where at least one of $n$ and $m$ equals one. The first part of section 3 is devoted to study the case where $p$ and $q$ are both even, while the second part of section 3 is devoted to study the case where $p$ and $q$ are both odd. An in-depth investigation of the cohomology ring structure of the space $S^{n-1} \times S^{m-1} / \mathcal{G}$ is needed. In addition, the exact values of the L-S category of $S^{n-1} \times S^{m-1} / \mathcal{G}$ in some special cases are obtained. Finally, in section 4 , we analyze the features of the function $f_{\mathcal{A}}$ when $p$ and $q$ are both odd, and introduce the Condition (S). Although Lemma 4.2 is very simple, it is crucial in computing the category of certain level sets of $f_{\mathcal{A}}$. Our main Theorem 1.3 is deduced from this lemma and the Liusternik- Schnirelmann multiplicity theorem.

\section{Singular values of a real tensor}

We assume $p, q, m$, and $n$ are positive integers with $n, m \geq 1$. Let $\mathcal{A}=\left(a_{i_{1} \cdots i_{p} j_{i} \cdots j_{q}}\right)$ be a real tensor, where $i_{k} \in\{1, \cdots, n\}$ for $1 \leq k \leq p$ and $j_{k} \in\{1, \cdots, m\}$ for $1 \leq k \leq q$. We call it a $(p, q)$ order $(n, m)$ dimensional tensor. When $p=q=1$, such a tensor $\mathcal{A}$ is simply an $n \times m$ rectangular matrix.

For any vector $x \in \mathbb{R}^{n}$ and $y \in \mathbb{R}^{m}$, let $\mathcal{A} x^{p-1} y^{q}$ be a vector in $\mathbb{R}^{n}$ with

$$
\left(\mathcal{A} x^{p-1} y^{q}\right)_{i}=\sum_{i_{2}, \cdots, i_{p}=1}^{n} \sum_{j_{1}, \cdots, j_{q}=1}^{m} a_{i i_{2} \cdots i_{p} j_{i} \cdots j_{q}} x_{i_{2}} \cdots x_{i_{p}} y_{j_{1}} \cdots y_{j_{q}} .
$$

Similarly, let $\mathcal{A} x^{p} y^{q-1}$ be a vector in $\mathbb{R}^{m}$ with

$$
\left(\mathcal{A} x^{p} y^{q-1}\right)_{j}=\sum_{i_{1}, \cdots, i_{p}=1}^{n} \sum_{j_{2}, \cdots, j_{q}=1}^{m} a_{i_{1} \cdots i_{p} j j_{2} \cdots j_{q}} x_{i_{1}} \cdots x_{i_{p}} y_{j_{2}} \cdots y_{j_{q}} .
$$

For any vectors $x \in \mathbb{R}^{n}, y \in \mathbb{R}^{m}$, and any real number $\alpha$, denote $x^{[\alpha]}=\left[x_{1}^{\alpha}, x_{2}^{\alpha}, \ldots, x_{n}^{\alpha}\right]^{T}$ and $y^{[\beta]}=\left[y_{1}^{\beta}, y_{2}^{\beta}, \cdots, y_{m}^{\beta}\right]^{T}$.

Definition 2.1. A complex number $\lambda$ is called a singular value of $\mathcal{A}$ if there exists a vector $(x, y) \in\left(\mathbb{C}^{n} \backslash\{0\}\right) \times\left(\mathbb{C}^{m} \backslash\{0\}\right)$, which is called the associate eigenvector, satisfying the following system:

$$
\begin{aligned}
& \mathcal{A} x^{p-1} y^{q}=\lambda x^{[M-1]} \\
& \mathcal{A} x^{p} y^{q-1}=\lambda y^{[M-1]}
\end{aligned}
$$

where $M=p+q$.

In the following, we restrict our attention to real singular values and real eigenvectors. We now present a way to reduce the singular value problem to an eigenvalue problem.

Let $N=n+m$ and let

$$
z=\left(\begin{array}{l}
x \\
y
\end{array}\right) \in \mathbb{R}^{N}=\mathbb{R}^{n} \times \mathbb{R}^{m}
$$

We introduce the lifting $\mathcal{T}_{\mathcal{A}}=\left(t_{k_{1}, k_{2}, \cdots, k_{M}}\right)$ of $\mathcal{A}$ as the $M=p+q$ order $N=m+n$ 
dimensional tensor defined by:

$$
t_{k_{1}, k_{2}, \cdots, k_{M}}=\left\{\begin{array}{l}
p a_{k_{1}, \cdots, k_{p}, k_{p+1}-n, \cdots, k_{M}-n}, \text { if }\left\{\begin{array}{c}
1 \leq k_{1}, \cdots, k_{p} \leq n, \\
n+1 \leq k_{p+1}, \cdots, k_{M} \leq N,
\end{array}\right. \\
q a_{k_{1}-m, \cdots, k_{q}-m, k_{q+1}, \cdots, k_{M}}, \text { if }\left\{\begin{array}{l}
m+1 \leq k_{1}, \cdots, k_{q} \leq N, \\
1 \leq k_{q+1}, \cdots, k_{M} \leq m,
\end{array}\right. \\
0, \text { elsewhere. }
\end{array}\right.
$$

By definition,

$$
\mathcal{T}_{\mathcal{A}} z^{M}=M \mathcal{A} x^{p} y^{q} \quad \text { where } z=(x, y), \quad \nabla\left(\mathcal{T}_{\mathcal{A}} z^{M}\right)=M\left(\begin{array}{l}
p \mathcal{A} x^{p-1} y^{q} \\
q \mathcal{A} x^{p} y^{q-1}
\end{array}\right),
$$

and

$$
\mathcal{T}_{\mathcal{A}} z^{M-1}=\left(\begin{array}{c}
p \mathcal{A} x^{p-1} y^{q} \\
q \mathcal{A} x^{p} y^{q-1}
\end{array}\right)
$$

Recall the definition of a weakly symmetric tensor given in [4]:

Definition 2.2. Let $\mathcal{C}$ be an $M$ order $N$ dimensional real tensor. It is called weakly symmetric if

$$
\nabla f(z)=M \mathcal{C} z^{M-1}, \quad \text { where } \quad f(z)=\mathcal{C} z^{M}, z \in \mathbb{R}^{M}
$$

Thus, $\mathcal{T}=\mathcal{T}_{\mathcal{A}}$ is weakly symmetric. Let $\mathcal{B}=\left(b_{k_{1}, \cdots, k_{M}}\right)$, where

$$
b_{k_{1}, k_{2}, \cdots, k_{M}}=\left\{\begin{array}{l}
p, \text { if } 1 \leq k_{1}=\cdots=k_{M} \leq n \\
q, \text { if } n+1 \leq k_{1}=\cdots=k_{M} \leq N \\
0, \text { elsewhere }
\end{array}\right.
$$

Since

$$
\mathcal{B} z^{M}=p \sum_{1}^{n} x_{i}^{M}+q \sum_{1}^{m} y_{j}^{M} \quad \text { and } \quad \nabla\left(\mathcal{B} z^{M}\right)=M\left(\begin{array}{c}
p x^{[M-1]} \\
q y^{[M-1]}
\end{array}\right)=M \mathcal{B} z^{M-1},
$$

the critical points of the function $f(z)=\mathcal{T} z^{M}=M \mathcal{A} x^{p} y^{q}$ with constraint $b(z)=\mathcal{B} z^{M}=$ 1 are solutions of the system:

$$
\begin{aligned}
& \mathcal{A} x^{p-1} y^{q}=\lambda x^{[M-1]} \\
& \mathcal{A} x^{p} y^{q-1}=\lambda y^{[M-1]}
\end{aligned}
$$

with $\lambda=\mathcal{T} z^{M}=M \mathcal{A} x^{p} y^{q}$.

Note that the only difference between the singular value of $\mathcal{A}$ and the eigenvalue of $\mathcal{T}$ is the following: in the definition of singular value, $x \neq 0$ and $y \neq 0$, but in the critical value case, $(x, y) \neq(0,0)$.

According to the definition, 0 is always a critical value of $f$ on $b^{-1}(1)$, because $(x, 0)$ with $p \sum_{1}^{n} x_{i}^{M}=1$, and $(0, y)$ with $q \sum_{1}^{m} y_{j}^{M}=1$, are critical points with critical value 0 , but they are not critical points with respect to any singular value. These critical points are trivial.

On the other hand, if $c \neq 0$ is an eigenvalue of $\mathcal{T}=\mathcal{T}_{\mathcal{A}}$, then it must be a singular value of $\mathcal{A}$, because if $(x, y)$ is a critical point with respect to $c$, then neither $x$ nor $y$ can be zero. 
Let $\mathbf{Z}$ be the set of all $(x, y) \in\left(\mathbb{R}^{n} \backslash\{0\}\right) \times\left(\mathbb{R}^{m} \backslash\{0\}\right)$ satisfying

$$
\begin{aligned}
& \mathcal{A} x^{p-1} y^{q}=0 \\
& \mathcal{A} x^{p} y^{q-1}=0 .
\end{aligned}
$$

Then $c=0$ is a singular value if and only if $\mathbf{Z} \neq \emptyset$. [4]:

The following multiplicity result for weakly symmetric tensor was established in

ThEOREM 2.3. Assume $\mathcal{A}$ is a weakly symmetric tensor, $\mathcal{B}$ is a weakly symmetric positive definite tensor, and both have the same order $M$ and dimension $N$. Then the eigenvalue problem $(\mathcal{A}-\lambda \mathcal{B}) z^{m}=0$ has at least $N$ real eigenvalues, counting multiplicity, with $N$ distinct pairs of real eigenvectors.

This theorem nevertheless does not help counting the multiplicities of singular values; as we have seen, there are a large number of trivial critical points. Regardless of this fact, we have:

TheOrem 2.4. Assume $M=p+q$ is even. Then for every nonzero tensor $\mathcal{A}$, the singular value always exists.

Proof. We consider the function $f(z)$ on the manifold $\mathbf{M}=\left\{z \in \mathbb{R}^{M} \mid b(z)=1\right\}$. Since $M$ is even, $\mathbf{M}$ is compact. Since $f \neq 0$, at least one of the maximum and the minimum value of $f$ does not equal zero, which is a nonzero critical value for $f$, so it is a nonzero eigenvalue for $\mathcal{T}_{\mathcal{A}}$, hence a singular value for $\mathcal{A}$.

REMARK 2.5. The result of Theorem 2.4 has been previously obtained in [5]. $M=$ $p+q$ being even is necessary to insure the compactness of the manifold $\mathbf{M}$. So, in our subsequent arguments, we need only distinguish the two cases where either $p$ and $q$ are both even or $p$ and $q$ are both odd.

Now we focus on the study of the multiplicities of singular values of $\mathcal{A}$. Observing that the functions $\mathcal{T} z^{M}$ and $\mathcal{B} z^{M}$ are all positively homogenous of total degree $M$, we normalize the vectors $x$ and $y$ by defining the following manifold:

$$
X=\left\{z=(x, y) \mid \sum_{1}^{n} x_{i}^{M}=\sum_{1}^{m} y_{j}^{M}=1\right\} .
$$

THEOREM 2.1. A vector $(x, y) \in\left(\mathbb{R}^{n} \backslash\{0\}\right) \times\left(\mathbb{R}^{m} \backslash\{0\}\right)$ is an eigenvector with respect to a singular value $\lambda$ of $\mathcal{A}$ if and only if

1. there is a unique $t>0$ such that $\lambda$ is an eigenvalue of $\mathcal{T}_{\mathcal{A}}$ with eigenvector $t(x, y) \in X$, when $\lambda \neq 0$,

2. there exist unique $s>0, t>0$, such that $\lambda$ is an eigenvalue of $\mathcal{T}_{\mathcal{A}}$ with eigenvector $(s x, t y) \in X$ when $\lambda=0$.

Proof. We note that $(x, y) \in X$ is a critical point of $f$ if and only if $(x, y) \in X$ satisfying (3) with critical value $\lambda=\mathcal{A} x^{p} y^{q}$. Also, $(x, y) \in\left(\mathbb{R}^{n} \backslash\{0\}\right) \times\left(\mathbb{R}^{m} \backslash\{0\}\right)$ is an eigenvector with singular value $\lambda=\frac{\mathcal{A} x^{p} y^{q}}{\Sigma_{1}^{n} x_{i}^{M}}=\frac{\mathcal{A} x^{p} y^{q}}{\Sigma_{1}^{m} y_{j}^{M}}$, which is equivalent to

$$
\sum_{1}^{n} x_{i}^{M}=\sum_{1}^{m} y_{j}^{M}=\frac{\mathcal{A} x^{p} y^{q}}{\lambda}
$$

if $\lambda \neq 0$. Let $t=\left(\frac{\lambda}{\mathcal{A} x^{p} y^{q}}\right)^{\frac{1}{M}}$ and the result follows. 
In the case $\lambda=0$, if $(x, y) \in X$ is a critical point with critical value $\lambda$, then it is obviously an eigenvector with singular value 0 . Conversely, if $(x, y)$ is an eigenvector with singular value 0 , then one may choose $s>0, t>0$ such that $(s x, t y) \in X$ satisfying (4).

The case when at least one of $n, m$ equals one is rather special. Without loss of generality, we assume that $m \geq n=1$. First, if $m=n=1, \mathcal{A}=a \neq 0$ is a scalar, and then

(i) for $p, q$ both even and $\lambda=a$, there are 2 pairs of eigenvectors $(1, \pm 1),(-1, \pm 1)$.

(ii) for $p, q$ both odd and $\lambda= \pm a$, there are 2 pairs of eigenvectors $\pm(1,1)$ corresponding to $\lambda=a$ and $\pm(-1,1)$ corresponding to $\lambda=-a$.

Next, if $m>n=1$, the $(p, q)$-order tensor $\mathcal{A}$ can also be seen as a $q$-order tensor. If there is no confusion, we shall not distinguish these two.

Corollary 2.6. Suppose that $M=p+q$ is even and $m>n=1$. If $\mathcal{T}_{\mathcal{A}}$ has, counting multiplicities, s nonzero eigenvalues and if the q-order tensor $\mathcal{A}$ has $r$ pairs of eigenvectors with eigenvalue zero, then $\mathcal{A}$ has, counting multiplicities, $r+s$ singular values.

In the rest of this paper, we may always assume $m, n \geq 2$.

\section{Proofs of Theorems 1.1, 1.2 and related results}

3.1. Some algebraic topological preliminaries. We have reduced the singular value problem to a critical point problem on the compact manifold $X=\{z=$ $\left.(x, y) \mid \sum_{1}^{n} x_{i}^{M}=\sum_{1}^{m} y_{j}^{M}=1\right\}$. We define a homeomorphism $X \simeq S^{n-1} \times S^{m-1}$ by

$$
\begin{aligned}
& x_{i}^{\prime}=\left|x_{i}\right|^{\frac{M}{2}} x_{i}, \quad i=1, \cdots n \\
& y_{j}^{\prime}=\left|y_{j}\right|^{\frac{M}{2}} y_{j}, \quad j=1, \cdots, m .
\end{aligned}
$$

Based on critical point theory, the minimum number of critical points of a function depends on the underlying topological space as well as the behavior of the given function. We shall study the singular value problem in several different cases.

The crucial result we rely on is the following classical Lusternik-Schnirelmann (L-S) Multiplicity Theorem; see for instance [6]:

TheOrem 3.1. Let $M$ be a compact $C^{1}$ manifold and suppose $f \in C^{1}\left(M, \mathbb{R}^{1}\right)$. Then the number of critical points of $f$ is greater than or equal to $\operatorname{cat}(M)$, where cat $(M)$ is the $L$-S category of $M$.

We begin by recalling some definitions and facts involving the L-S category of a topological space; for details, see $[6,10,11,23]$ :

Proposition 3.2. Let $X$ and $Y$ be paracompact, Hausdorff, and path-connected topological spaces.

1. $\operatorname{cat}(X)$ is defined to be the least integer $k$ such that $\exists$ open covering $V_{1}, V_{2}, \ldots, V_{k}$ of $X$, where $V_{i}, 1 \leq i \leq k$ are contractible open subsets of $X$. In particular, $\operatorname{cat}\left(S^{a}\right)=2$ and $\operatorname{cat}\left(\mathbb{R} \mathbb{P}^{a}\right)=a+1$.

2. $\operatorname{cup}^{\mathrm{h}}(X)$, the $\mathbb{Z}_{2}$ cup-length of $X$, is defined to be the least integer $k$ such that all $k+1$ fold cup products vanish in the cohomology ring $H^{*}\left(X ; \mathbb{Z}_{2}\right)$. 
3.

$$
\operatorname{cup}^{\mathrm{h}}(X)+1 \leq \operatorname{cat}(X) \leq \operatorname{dim}(X)+1
$$

and

$$
\operatorname{cup}^{\mathrm{h}}(X \times Y)+1 \leq \operatorname{cat}(X \times Y) \leq \operatorname{cat}(X)+\operatorname{cat}(Y)-1 .
$$

4. Let $\beta \in H^{n}\left(S^{a} ; \mathbb{Z}_{2}\right)$ be the Kronnecker dual of the fundamental class (mod 2 orientation class). One has:

$$
H^{*}\left(S^{a} ; \mathbb{Z}_{2}\right)=\mathbb{Z}_{2}[\beta] / \beta^{2}=0 . \quad \text { So, } \quad \operatorname{cup}^{\mathrm{h}}\left(S^{a}\right)=1 .
$$

5. Let $\mathbb{Z}_{2}$ denote the two element group consisting of the identity map and the antipodal map on the space $\mathbb{R}^{a+1} \backslash\{0\}$. Let $\mathbb{R P}^{a}=S^{a} / \mathbb{Z}_{2}$ be the real projective space, and let $w_{1} \in H^{1}\left(\mathbb{R P}^{a} ; \mathbb{Z}_{2}\right)$ be the first Stiefel-Whitney class of the classifying line bundle over $\mathbb{R} \mathbb{P}^{n}$. One has $H^{*}\left(\mathbb{R P}^{a} ; \mathbb{Z}_{2}\right)$ is isomorphic to the truncated $\mathbb{Z}_{2}$-polynomial algebra $\mathbb{Z}_{2}\left[w_{1}\right] / w_{1}^{a+1}=0$. Hence, $\operatorname{cup}^{\mathrm{h}}\left(\mathbb{R P}^{a}\right)=a$.

6. If there exists a discrete group $\pi$ acting freely on $X$, then $\operatorname{cat}(X) \leq \operatorname{cat}(X / \pi)$.

7. If $X$ is a connected $P L$-manifold satisfies $\operatorname{cat}(X) \geq(\operatorname{dim}(X)+3) / 2$, then $\operatorname{cat}\left(X \times S^{\ell}\right)=\operatorname{cat}(X)+1$ for all $\ell \geq 1$, i.e., the Ganea conjecture on $L$-S category holds for $X$.

Some more results in algebraic topology concerning the cup-product in product spaces are needed. We refer to [24] for details.

Theorem 3.3. Let $R$ be a field. In the graded cohomology $R$-algebra $H^{*}(X \times Y ; R)$, we have the following product formula:

$$
\left(u_{1} \times v_{1}\right) \cup\left(u_{2} \times v_{2}\right)=(-1)^{\left|v_{1}\right|\left|u_{2}\right|}\left(u_{1} \cup u_{2}\right) \times\left(v_{1} \cup v_{2}\right),
$$

where $\left|v_{1}\right|$ and $\left|u_{2}\right|$ denote the degrees of the cohomology classes of $v_{1}$ and $u_{2}, \cup$ denotes the standard cohomology cup products, and $\times$ denotes the standard cohomology cross product, i.e. if $\pi_{1}: X \times Y \rightarrow X$ and $\pi_{2}: X \times Y \rightarrow Y$ are the projections, then for any $u \in H^{*}(X ; R)$ and $v \in H^{*}(Y ; R)$,

$$
u \times v=\pi_{1}^{*}(u) \cup \pi_{2}^{*}(v) .
$$

Note: if $R$ has characteristic 2, then there is no sign change in the product formula.

3.2. When $\mathbf{p}$ and $\mathbf{q}$ are both even. We first consider the case where $p$ and $q$ are both even. In this case, the function $f_{\mathcal{A}}(x, y)=\mathcal{A} x^{p} y^{q}$ can be seen as a function defined on the product space $\mathbb{R} \mathbb{P}^{n-1} \times \mathbb{R} \mathbb{P}^{m-1}=S^{n-1} \times S^{m-1} / \mathbb{Z}_{2} \oplus \mathbb{Z}_{2}$, where the $\mathbb{Z}_{2} \oplus$ $\mathbb{Z}_{2}$ action is give by the equivalence relation $(x, y) \sim(-x, y) \sim(x,-y) \sim(-x,-y)$.

By setting $X:=\mathbb{R} \mathbb{P}^{n-1}$ and $Y:=\mathbb{R} \mathbb{P}^{m-1}$ for $m, n \geq 2$, we have the following identities:

ThEOREM 3.4. $\quad \operatorname{cup}^{\mathrm{h}}\left(\mathbb{R P}^{n-1} \times \mathbb{R} \mathbb{P}^{m-1}\right)=n+m-2$ and $\operatorname{cat}\left(\mathbb{R P}^{n-1} \times \mathbb{R P}^{m-1}\right)=n+$ $m-1$.

Proof. It suffices to show $\operatorname{cup}^{\mathrm{h}}\left(\mathbb{R P}^{n-1} \times \mathbb{R P}^{m-1}\right)=n+m-2$, since the rest will follow from

$$
\operatorname{cat}\left(\mathbb{R} \mathbb{P}^{n-1} \times \mathbb{R} \mathbb{P}^{m-1}\right) \leq \operatorname{dim}\left(\mathbb{R} \mathbb{P}^{n-1} \times \mathbb{R} \mathbb{P}^{m-1}\right)+1=n+m-1 .
$$


Let $a \in H^{1}\left(\mathbb{R P}^{n-1} ; \mathbb{Z}_{2}\right)$ and $b \in H^{1}\left(\mathbb{R P}^{m-1} ; \mathbb{Z}_{2}\right)$ be the corresponding generators. For $0 \leq i \leq n-1$ and $0 \leq j \leq m-1, a^{i} \in H^{i}\left(\mathbb{R} P^{n-1} ; \mathbb{Z}_{2}\right)$ and $b^{j} \in H^{j}\left(\mathbb{R} \mathbb{P}^{m-1} ; \mathbb{Z}_{2}\right)$ are the respective generators. By Künneth's Formula, $a^{i} \times b^{j}$ is a generator of $H^{i+j}\left(\mathbb{R P}^{n-1} \times\right.$ $\left.\mathbb{R} \mathbb{P}^{m-1} ; \mathbb{Z}_{2}\right)$ for $0 \leq k=i+j \leq m+n-2$. In the cohomology algebra $H^{*}\left(\mathbb{R P}^{n-1} \times\right.$ $\left.\mathbb{R P}^{m-1} ; \mathbb{Z}_{2}\right)$, consider the $(n+m-2)$-fold cup product

$$
(a \times 1)^{n-1} \cup(1 \times b)^{m-1}=\left(a^{n-1} \times 1\right) \cup\left(1 \times b^{m-1}\right)=a^{n-1} \times b^{m-1} \neq 0,
$$

so $\operatorname{cup}^{\mathrm{h}}\left(\mathbb{R} \mathbb{P}^{n-1} \times \mathbb{R} \mathbb{P}^{m-1}\right) \geq n+m-2$. On the other hand, $\operatorname{cup}^{\mathrm{h}}\left(\mathbb{R} \mathbb{P}^{n-1} \times \mathbb{R} \mathbb{P}^{m-1}\right) \leq$ $n+m-2$ by (3) in the proposition, which completes the proof.

We can now derive, by an inductive argument, the following generalization:

Corollary 3.5. Let $n_{1}, \cdots, n_{k} \geq 1$. Then

$\operatorname{cup}^{\mathrm{h}}\left(\mathbb{R} \mathbb{P}^{n_{1}} \times \cdots \times \mathbb{R} \mathbb{P}^{n_{k}}\right)=n_{1}+\cdots+n_{k}$ and $\operatorname{cat}\left(\mathbb{R} \mathbb{P}^{n_{1}} \times \cdots \times \mathbb{R} \mathbb{P}^{n_{k}}\right)=n_{1}+\cdots+n_{k}+1$

Combining Theorems 3.1 and 3.4, we arrive at:

TheOrem 3.6. Let $\mathcal{A}$ be a $(p, q)$ order $(n, m)$ dimension real tensor. Assume both $p$ and $q$ are even; then $\mathcal{A}$ possesses at least $m+n-1$ singular values, counting multiplicities.

3.3. When $\mathbf{p}$ and $\mathbf{q}$ are both odd. The rest of this section is focused on the case where $p$ and $q$ are both odd. We shall assume $m \geq n \geq 2$ unless otherwise specified. For notational simplicity, we make the following dimensional shift.

Let $\mathcal{G}$ be the group consisting of two elements: $\{\mathrm{id}, g\}$, where id is the identity map and $g:(x, y) \mapsto(-x,-y)$ is the antipodal map. Let $M_{n, m}=S^{n} \times S^{m} / \mathcal{G}$ be the quotient manifold with the quotient map $p_{1}$.

THEOREM 3.7. The following properties hold for $H^{*}\left(M_{n, m} ; \mathbb{Z}_{2}\right)$ :

1. There is an isomorphism $\mathrm{J}$ between the cohomology groups

$$
\jmath: H^{*}\left(M_{n, m} ; \mathbb{Z}_{2}\right) \stackrel{\cong}{\rightrightarrows} H^{*}\left(\mathbb{R} P^{n} ; \mathbb{Z}_{2}\right) \otimes_{\mathbb{Z}_{2}} H^{*}\left(S^{m} ; \mathbb{Z}_{2}\right) .
$$

2. The cohomology algebra $H^{*}\left(M_{n, m} ; \mathbb{Z}_{2}\right)$ has $H^{*}\left(\mathbb{R P}^{n} ; \mathbb{Z}_{2}\right) \cong \mathbb{Z}_{2}\left[w_{1}\right] / w_{1}^{n+1}=0$ as a subalgebra.

3. $\operatorname{cat}\left(M_{n, m}\right) \geq \operatorname{cup}^{\mathrm{h}}\left(M_{n, m}\right)+1 \geq \min \{m, n\}+2$.

Proof. Let $i: S^{n} \hookrightarrow S^{m}$ be the standard embedding. Let $\Delta: S^{n} \rightarrow S^{n} \times S^{n}$ be the diagonal map, i.e., $\Delta: x \mapsto(x, x)$. Let $\phi=(1 \times i) \circ \Delta$ be the composition, where 1 represents the identity map on the first factor; then $\phi: S^{n} \rightarrow S^{n} \times S^{m}$ is a $\mathbb{Z}_{2}$-equivariant embedding. We identify $\mathbb{R} \mathbb{P}^{n}$ with $S^{n} / \mathbb{Z}_{2}$ under the projection map $p_{2}$, where the $\mathbb{Z}_{2}$-action is given by gluing the antipodal points $x \sim-x$. It follows that $\phi$ induces a smooth embedding $\eta: \mathbb{R P}^{n} \rightarrow M_{n, m}$.

Let $\mathbb{L}$ be the classifying line bundle over $\mathbb{R P}^{n}$. Let $E=(m+1) \cdot \mathbb{L}$ be the Whitney sum of $m+1$ copies of $\mathbb{L}$, which is itself an $(m+1)$-plane bundle over $\mathbb{R} \mathbb{P}^{n}$ with the canonical projection $\pi$. We identify:

$$
(m+1) \cdot \mathbb{L} \cong S^{n} \times \mathbb{R}^{m+1} / \mathcal{G} .
$$

Let $E_{0}$ be the complement of the zero section in the vector bundle $E$. Let $\pi_{0}$ be the restriction of $\pi$ on $E_{0}$. Since $M_{n, m}$ is a strong deformation retraction of $E_{0}$, they 
are homotopy equivalent, i.e. there exist maps $r: E_{0} \rightarrow M_{n, m}$ and $s: M_{n, m} \rightarrow E_{0}$, such that $r \circ s=\operatorname{id}_{M_{n, m}}$ and $s \circ r=\operatorname{id}_{E_{0}}$. Let $\zeta=s \circ \eta: \mathbb{R} \mathbb{P}^{n} \rightarrow E_{0}$ be the composite map.

We now illustrate the relations among these spaces and the corresponding maps in the following commutative diagrams:

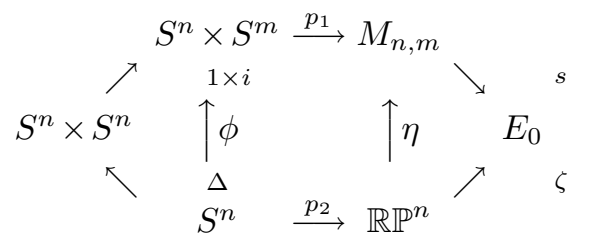

and

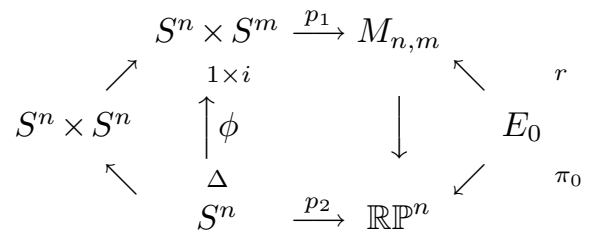

Since $M_{n, m}$ is homotopy equivalent to $E_{0}$, they have isomorphic cohomology rings. In the subsequent arguments concerning the mod-2 cohomology algebra, we shall use $E_{0}$ in place of $M_{n, m}$. Note that $E_{0}$ is a $S^{m}$-bundle over $\mathbb{R P}^{n}$, thus we have the following mod-2 Gysin cohomology long exact sequence:

$$
\cdots \stackrel{\psi}{\longrightarrow} H^{j-m-1}\left(\mathbb{R P}^{n} ; \mathbb{Z}_{2}\right) \stackrel{\mu}{\longrightarrow} H^{j}\left(\mathbb{R P}^{n} ; \mathbb{Z}_{2}\right) \stackrel{\pi_{0}^{*}}{\longrightarrow} H^{j}\left(E_{0} ; \mathbb{Z}_{2}\right) \stackrel{\psi}{\longrightarrow} H^{j-m}\left(\mathbb{R P}^{n} ; \mathbb{Z}_{2}\right) \stackrel{\mu}{\longrightarrow} \cdots
$$

where $\mu: x \mapsto x \cup w_{m+1}$ with $w_{m+1} \in H^{m+1}\left(\mathbb{R P}^{n} ; \mathbb{Z}_{2}\right)$ being the top Stiefel-Whitney class of $E$. For details, see [16]. Since $m \geq n, w_{m+1} \in H^{m+1}\left(\mathbb{R P}^{n} ; \mathbb{Z}_{2}\right)=0$, so the map $\mu$ in the Gysin sequence $(\mathrm{G})$ is in fact the zero homomorphism. Consequently, the Gysin sequence $(\mathrm{G})$ breaks up and yields isomorphisms

$$
H^{j+m}\left(E_{0} ; \mathbb{Z}_{2}\right) \cong H^{j}\left(\mathbb{R} \mathbb{P}^{n} ; \mathbb{Z}_{2}\right)
$$

if either $m>n$ and $j=0,1, \cdots, n$ or $m=n$ and $j=1, \cdots, n$. Furthermore, we claim there exists a unique nonzero class $v \in H^{m}\left(E_{0} ; \mathbb{Z}_{2}\right)$ satisfying $\delta^{*}(v)=u$, where $\delta^{*}$ is the co-boundary homomorphism in the cohomology long exact sequence of the pair $\left(E, E_{0}\right)$ and $u \in H^{m+1}\left(E, E_{0} ; \mathbb{Z}_{2}\right)$ denotes the Thom class. For $m>n$, from $H^{m}\left(E ; \mathbb{Z}_{2}\right)=H^{m+1}\left(E ; \mathbb{Z}_{2}\right)=0, \delta^{*}: H^{m}\left(E_{0} ; \mathbb{Z}_{2}\right) \rightarrow H^{m+1}\left(E, E_{0} ; \mathbb{Z}_{2}\right)$ is an isomorphism, thus the existence and uniqueness of $v$ is clear. For $m=n$, since $H^{m}\left(E, E_{0} ; \mathbb{Z}_{2}\right)=0$ and $H^{m+1}\left(E, E_{0} ; \mathbb{Z}_{2}\right)=\{u\} \cdot \mathbb{Z}_{2}$, again there exists a unique class $v \in H^{m}\left(E_{0} ; \mathbb{Z}_{2}\right)$, such that $\delta^{*}(v)=u$ and $H^{m}\left(E_{0} ; \mathbb{Z}_{2}\right) \cong H^{m}\left(\mathbb{R} \mathbb{P}^{n} ; \mathbb{Z}_{2}\right) \oplus\{v\} \cdot \mathbb{Z}_{2}$. These together prove assertion (1).

We now turn our attention to the ring structure. Since we have defined the composite map $\zeta: \mathbb{R} \mathbb{P}^{n} \rightarrow E_{0}$, it induces the map $\zeta^{*}: H^{*}\left(E_{0} ; \mathbb{Z}_{2}\right) \rightarrow H^{*}\left(\mathbb{R} \mathbb{P}^{n} ; \mathbb{Z}_{2}\right)$ such that $\zeta^{*}$ is a multiplicative left inverse of $\pi_{0}^{*}$, i.e.,

$$
\zeta^{*} \circ \pi_{0}^{*}=1_{H^{*}\left(\mathbb{R}^{p} ; \mathbb{Z}_{2}\right)} .
$$

By the naturality of the cup product, both $\zeta^{*}: H^{*}\left(E_{0} ; \mathbb{Z}_{2}\right) \rightarrow H^{*}\left(\mathbb{R} \mathbb{P}^{n} ; \mathbb{Z}_{2}\right)$ and $\pi_{0}^{*}$ : $H^{*}\left(\mathbb{R} \mathbb{P}^{n} ; \mathbb{Z}_{2}\right) \rightarrow H^{*}\left(E_{0} ; \mathbb{Z}_{2}\right)$ preserve the cup product, i.e.,

$$
\zeta^{*}(a \cup b)=\zeta^{*}(a) \cup \zeta^{*}(b) \quad \text { and } \quad \pi_{0}^{*}(a \cup b)=\pi_{0}^{*}(a) \cup \pi_{0}^{*}(b) \quad \forall a, b .
$$


Let $\omega=\pi_{0}^{*} w_{1} \in H^{1}\left(E_{0} ; \mathbb{Z}_{2}\right)$, where $w_{1} \in H^{1}\left(\mathbb{R P}^{n} ; \mathbb{Z}_{2}\right)$ is the generator; we then have

$$
\pi_{0}^{*}\left(w_{1}^{i}\right)=\left(\pi_{0}^{*} w_{1}\right)^{i}=\omega^{i} \in H^{i}\left(E_{0} ; \mathbb{Z}_{2}\right) \quad \forall i .
$$

Since $\pi_{0}^{*}$ is monomorphic, we have

$$
0 \neq \omega^{i} \in H^{i}\left(E_{0} ; \mathbb{Z}_{2}\right), \quad 0 \leq i \leq n .
$$

By our first assertion, for $1 \leq i \leq n, H^{i}\left(E_{0} ; \mathbb{Z}_{2}\right) \cong H^{i}\left(\mathbb{R P}^{n} ; \mathbb{Z}_{2}\right)$ has only one generator $\omega^{i}$, thus, $H^{i}\left(E_{0} ; \mathbb{Z}_{2}\right)$ is generated by $\left\{\omega^{i}\right\}$; assertion (2) follows.

Although all maps in the Gysin sequence $(\mathrm{G})$ are module homomorphisms, the map $\psi$ has certain multiplicative property. We employ the following result due to $\mathrm{W}$. Massey (Lemma 1 in [15]): If $x \in H^{p}\left(\mathbb{R P}^{n} ; \mathbb{Z}_{2}\right)$ and $y \in H^{q}\left(E_{0} ; \mathbb{Z}_{2}\right)$, then $\psi\left[\left(\pi_{0}^{*} x\right) \cup y\right]=$ $x \cup \psi(y)$. Choose $x=w_{1}^{n} \in H^{n}\left(\mathbb{R}^{n} ; \mathbb{Z}_{2}\right)$, and $y=v \in H^{m}\left(E_{0} ; \mathbb{Z}_{2}\right)$; we claim $\psi(v)=1$. If we set $j=m$ in $(\mathrm{G})$, then the 3 -term sequence

$$
0 \rightarrow H^{m}\left(\mathbb{R} \mathbb{P}^{n} ; \mathbb{Z}_{2}\right) \stackrel{\pi_{0}^{*}}{\longrightarrow} H^{m}\left(E_{0} ; \mathbb{Z}_{2}\right) \stackrel{\psi}{\longrightarrow} H^{0}\left(\mathbb{R} \mathbb{P}^{n} ; \mathbb{Z}_{2}\right) \rightarrow 0
$$

is exact. Note that $H^{0}\left(\mathbb{R} \mathbb{P}^{n} ; \mathbb{Z}_{2}\right)=\{1\} \cdot \mathbb{Z}_{2}$, where 1 denotes the unit in $H^{*}\left(\mathbb{R} \mathbb{P}^{n} ; \mathbb{Z}_{2}\right)$.

If $m>n$, then $H^{m}\left(\mathbb{R P}^{n} ; \mathbb{Z}_{2}\right)=0$ and it follows that $\psi(v)=1$. If $m=n$, from the exact sequence

$$
H^{m}\left(\mathbb{R} \mathbb{P}^{n} ; \mathbb{Z}_{2}\right) \rightarrow H^{m}\left(E_{0} ; \mathbb{Z}_{2}\right) \cong H^{m}\left(\mathbb{R} \mathbb{P}^{n} ; \mathbb{Z}_{2}\right) \oplus\{v\} \cdot \mathbb{Z}_{2} \rightarrow H^{0}\left(\mathbb{R} \mathbb{P}^{n} ; \mathbb{Z}_{2}\right)
$$

again we have $\psi(v)=1$. The image $\psi\left[\left(\pi_{0}^{*} w_{1}^{n}\right) \cup v\right]=w_{1}^{n} \cup \psi(v)=w_{1}^{n} \neq 0 \quad$ in $H^{n}\left(\mathbb{R P}^{n} ; \mathbb{Z}_{2}\right)$. This shows $\operatorname{cup}^{\mathrm{h}}\left(E_{0}\right) \geq n+1$, hence it completes our proof.

We denote the category of the space $M_{n, m}$ by cat $\mathcal{G}$, and simply written as cat. Applying the Liusternik Schnirelmann Theorem directly, we obtain:

Theorem 3.8. Let $\mathcal{A}$ be a $(p, q)$ order $(n, m)$ dimension real tensor. Assume that $p$ and $q$ are both odd. Then there exist singular values with at least $\min (n, m)+1$ pairs of eigenvectors for $\mathcal{A}$.

Proof. Under the $\mathcal{G}$-action, the function $f_{\mathcal{A}}(x, y)=\mathcal{A} x^{p} y^{q}$ on $S^{n-1} \times S^{m-1}$ defines a function $\hat{f_{\mathcal{A}}}$ on $M_{n-1, m-1}$. By Theorem 3.6 (3), $\operatorname{cat}\left(M_{n-1, m-1}\right) \geq$ $\operatorname{cup}^{\mathrm{h}}\left(M_{n-1, m-1}\right)+1 \geq \min (n, m)+1$. Therefore, $\hat{f}_{A}$ has at least $\min (n, m)+1 \mathcal{G}$ critical orbits, thus $f_{\mathcal{A}}$ has singular values with as least $\min (n, m)+1$ pairs of eigenvectors.

REMARK 3.9. Utilizing another result in the same paper [15], one can actually prove that the cohomology ring $H^{*}\left(E_{0} ; \mathbb{Z}_{2}\right)$ is a quadratic extension of the cohomology ring $H^{*}\left(\mathbb{R P}^{n} ; \mathbb{Z}_{2}\right)$. In particular, given any element $u \in H^{q}\left(E_{0} ; \mathbb{Z}_{2}\right)$, there exist unique elements $u_{1} \in H^{q}\left(\mathbb{R}^{n} ; \mathbb{Z}_{2}\right)$ and $u_{2} \in H^{q-m}\left(\mathbb{R}^{n} ; \mathbb{Z}_{2}\right)$ such that $u=\pi_{0}^{*}\left(u_{1}\right)+a \cdot \pi_{0}^{*}\left(u_{2}\right)$.

3.4. Corollaries and remarks. First, we prove the following Borsuk-Ulam type theorem:

Lemma 3.10. Let $p_{1}: S^{n} \times S^{m} \rightarrow M_{n, m}$ be the quotient map. Let $\sigma$ be a path connecting a pair of antipodal points $(x, y),(-x,-y) \in S^{n} \times S^{m}$. Then $p_{1}(\sigma)$ is a singular closed chain with a nonzero representation in $H_{1}\left(M_{n, m} ; \mathbb{Z}_{2}\right)$.

Proof. Let $\sigma$ be a path connecting a pair of antipodal points $(x, y),(-x,-y) \in$ $S^{n} \times S^{m}$. Since $\mathcal{G} \rightarrow S^{n} \times S^{m} \stackrel{p_{1}}{\longrightarrow} M_{n, m}$ is a fibration, $\pi_{1}\left(M_{n, m}\right) \cong \mathcal{G} \cong \mathbb{Z}_{2}$, so $p_{1}(\sigma)$ is a closed loop in $M_{n, m}$, which is not null-homotopic, i.e., the homotopy class $\left[p_{1}(\sigma)\right] \neq$ 
0 . Let $h: \pi_{1}\left(M_{n, m}\right) \rightarrow H_{1}\left(M_{n, m}\right)$ be the Hurewicz homomorphism. Since $M_{n, m}$ is path connected and $\pi_{1}\left(M_{n, m}\right) \cong \mathbb{Z}_{2}$ is commutative, $h: \pi_{1}\left(M_{n, m}\right) \rightarrow H_{1}\left(M_{n, m}\right)$ is an isomorphism and $h_{*}\left[p_{1}(\sigma)\right] \in H_{1}\left(M_{n, m}\right)$ is a generator, and hence is nonzero.

THEOREM 3.11. Let $f: S^{n} \times S^{m} \rightarrow S^{k} \times S^{\ell}$ be a continuous $\mathcal{G}$-equivariant map with $\ell>\max \{m, n\}$. Then $k \geq \min \{m, n\}$.

Proof. We argue by contradiction. Suppose $k<\min \{m, n\} . M_{n, m}$ and $M_{k, \ell}$ are $\mathcal{G}$-spaces via the quotient maps $p_{1}: S^{n} \times S^{m} \rightarrow M_{n, m}$ and $p_{1}^{\prime}: S^{k} \times S^{\ell} \rightarrow M_{k, \ell}$, respectively. The map $f$ induces a map: $\bar{f}: M_{n, m} \rightarrow M_{k, \ell}$ satisfying:

$$
\bar{f} \circ p_{1}=p_{1}^{\prime} \circ f \text {. }
$$

We claim the homomorphism

$$
\bar{f}_{*}: H_{1}\left(M_{n, m} ; \mathbb{Z}_{2}\right) \rightarrow H_{1}\left(M_{k, \ell} ; \mathbb{Z}_{2}\right)
$$

is nonzero. To see this, we choose a path $\sigma$ connecting a pair of antipodal points $(x, y), \quad(-x,-y) \in S^{n} \times S^{m}$, then $f \circ \sigma$ is a path connecting the antipodal points $f(x, y),-f(x, y)$ in $S^{k} \times S^{\ell}$. From the proceeding lemma, we see that $p_{1}^{\prime}(f \circ \sigma)$ is a singular closed chain, which has a nonzero representation in $H^{1}\left(M_{k, \ell} ; \mathbb{Z}_{2}\right)$. This means $\bar{f}_{*} \neq 0$. By duality, we have

$$
0 \neq \bar{f}^{*}: \mathbb{Z}_{2} \cong H^{1}\left(M_{k, \ell} ; \mathbb{Z}_{2}\right) \rightarrow H^{1}\left(M_{n, m} ; \mathbb{Z}_{2}\right) \cong \mathbb{Z}_{2}
$$

Since $H^{1}\left(M_{k, \ell} ; \mathbb{Z}_{2}\right)$ has only one generator $a, \bar{f}^{*}(a) \neq 0$. Also, $H^{1}\left(M_{n, m} ; \mathbb{Z}_{2}\right)$ has a unique generator $b$, thus $b=\bar{f}^{*}(a)$. According to Theorem 3.6 (2),

$$
0 \neq b^{n}=\bar{f}^{*}\left(a^{n}\right) .
$$

This implies $a^{n} \neq 0$ in $H^{n}\left(M_{k, \ell} ; \mathbb{Z}_{2}\right)$. Now $\ell>k$, so $k \geq n$. This is a contradiction.

In general, it is unclear whether Theorem 3.6 (3) is sharp; however, we can calculate the exact values of $\operatorname{cat}\left(M_{n, m}\right)$ in certain cases:

THEOREM 3.12. The following conclusions hold

1. $\operatorname{cat}\left(M_{1,1}\right)=3$.

2. $\operatorname{cat}\left(M_{2,2}\right)=4$.

3. $\operatorname{cat}\left(M_{3,3}\right)=5$.

Proof. By Theorem 3.6 (3), it suffices to show $\operatorname{cat}\left(M_{n, n}\right) \leq n+2$ for $1 \leq n \leq 3$. Assertion (1) follows from the inequalities:

$$
\operatorname{cat}\left(M_{1,1}\right) \leq \operatorname{dim}\left(M_{1,1}\right)+1=3 .
$$

Since $S^{2} \times S^{2}$ is diffeomorphic to $\tilde{G}_{2}\left(\mathbb{R}^{4}\right)$, the Grassmannian of oriented 2dimensional subspaces of $\mathbb{R}^{4}$, which is the universal double covering of $G_{2}\left(\mathbb{R}^{4}\right)$, the unoriented Grassmannian of 2-dimensional subspaces of $\mathbb{R}^{4}$ (page 20, ex.10 (c) [9]). So, $M_{2,2}$ is homeomorphic to $G_{2}\left(\mathbb{R}^{4}\right)$. Next we apply Berstein-Schwartz's Theorem. If $X$ is a closed, connected manifold with $\pi_{1}(X) \cong \mathbb{Z}_{2}$, then $\operatorname{cat}(X)=\operatorname{dim}(X)+1$ if and only if $\varphi^{\operatorname{dim}(X)} \neq 0$, where $\varphi$ generates $H^{1}\left(X ; \mathbb{Z}_{2}\right)$ (Prop. 5.4 in [10]) to this case with $\varphi=w_{1}$. A classical result due to Borel [1] reveals the cohomology ring $H^{*}\left(G_{2}\left(\mathbb{R}^{q}\right) ; \mathbb{Z}_{2}\right) \cong \mathbb{Z}_{2}\left[w_{1}, w_{2}\right] / w_{i}^{\perp}=0$ for $i \geq q-1$, where $w_{1}, w_{2}$ are the first and second Stiefel-Whitney classes of the universal 2-plane bundle $\gamma_{2}$ over $G_{2}\left(\mathbb{R}^{q}\right), \gamma_{2} \oplus \gamma_{2}^{\perp}$ is the trivial bundle of dimension $q$, and $w_{i}^{\perp}=w_{i}\left(\gamma_{2}^{\perp}\right)$. It is shown, in [25, Lem. 2.5] that $w_{q-1}^{\perp}=w_{1}^{q-1}$ if and only if $q$ is a power of 2 , consequently $w_{1}^{3}=0$ and $\operatorname{cat}\left(G_{2}\left(\mathbb{R}^{4}\right)\right)<5$, which proves $(2)$. 
Recall that $\operatorname{Spin}(n)$ is the universal double covering of $\mathrm{SO}(n)$ for $n \geq 3$ ([2, page 6, Prop. 6.19]). As special cases, since $\mathrm{SO}(3)$ is homeomorphic to $\mathbb{R P}^{3}$ (page 20, ex.10(b) [9]), $\operatorname{Spin}(3)=S^{3}$. Furthermore, $\operatorname{Spin}(4)=\operatorname{Spin}(3) \times \operatorname{Spin}(3)$ (page 292, ex. $12[2])$. So, $\operatorname{Spin}(4)=S^{3} \times S^{3}$ is the universal double covering of $\mathrm{SO}(4)$. It follows that $M_{33}=\mathrm{SO}(4)=\mathbb{R P}^{3} \times S^{3}$. Therefore, from the proposition (7) in Proposition 3.2, we obtain $\operatorname{cat}\left(M_{3,3}\right)=\operatorname{cat}\left(\mathbb{R P}^{3} \times S^{3}\right)=5$.

We end this section by exhibiting an example showing:

Proposition 3.13. $M_{n, m}$ is, in general, not homotopy equivalent to $\mathbb{R P}^{n} \times S^{m}$.

Proof. It suffices to consider the case $M_{2,2}$, which is homeomorphic to $G_{2}\left(\mathbb{R}^{4}\right)$. Since $G_{k}\left(\mathbb{R}^{n}\right)$ is orientable if and only if $n$ is even, $G_{2}\left(\mathbb{R}^{4}\right)$ is orientable. In particular, $H_{4}\left(G_{2}\left(\mathbb{R}^{4}\right)\right)=\mathbb{Z}$.

On the other hand, recall $H_{0}\left(\mathbb{R} \mathbb{P}^{2}\right)=\mathbb{Z}, H_{1}\left(\mathbb{R P}^{2}\right)=\mathbb{Z}_{2}$, and $H_{2}\left(\mathbb{R P}^{2}\right)=0$, so by the Künneth Formula, we have

$$
\begin{aligned}
H_{4}\left(\mathbb{R P}^{2} \times S^{2}\right) & =\sum_{i+j=4} H_{i}\left(\mathbb{R P}^{2}\right) \otimes H_{j}\left(S^{2}\right) \oplus \sum_{i+j=3} H_{i}\left(\mathbb{R P}^{2}\right) * H_{j}\left(S^{2}\right) \\
& =H_{2}\left(\mathbb{R} \mathbb{P}^{2}\right) * H_{1}\left(S^{2}\right) \\
& =\mathbb{Z}_{2} * \mathbb{Z}=0,
\end{aligned}
$$

where $*$ denotes the standard torsion product from homological algebra, i.e., $\mathbb{R P}^{2} \times S^{2}$ fails to be orientable. This completes the proof.

\section{Further multiplicity results}

In this section, we endeavor to improve the estimate given by Theorem 3.8. When both $p$ and $q$ are odd, Theorem 3.8 asserts that the function $f_{\mathcal{A}}(x, y)=\mathcal{A} x^{p} y^{q}$ has at least $\min (n, m)+1$ pairs of antipodal critical points on $S^{n-1} \times S^{m-1}$. However, it is worth noticing the additional special feature of the function $f_{\mathcal{A}}$ :

$$
f_{\mathcal{A}}(x, y)=f_{\mathcal{A}}(-x,-y)=-f_{\mathcal{A}}(-x, y)=-f_{\mathcal{A}}(x,-y)
$$

Thus, $c>0$ is a critical value with a pair of critical points $\left(x_{0}, y_{0}\right),\left(-x_{0},-y_{0}\right)$ if and only if $-c<0$ is a critical value with a pair of critical points $\left(-x_{0}, y_{0}\right),\left(x_{0},-y_{0}\right)$.

In the remainder of this section, we always assume $m \geq n$. We introduce the level subsets

$$
f_{a}:=\left\{(x, y) \in S^{n-1} \times S^{m-1} \mid f_{\mathcal{A}}(x, y) \leq a\right\}, \forall a \in \mathbb{R}^{1},
$$

and the 0 critical set $\Lambda$, i.e., the set $(x, y) \in S^{n-1} \times S^{m-1}$ of solutions of the system

$$
\left\{\begin{array}{l}
\mathcal{A} x^{p-1} y^{q}=0 \\
\mathcal{A} x^{p} y^{q-1}=0 .
\end{array}\right.
$$

They are $\mathcal{G}$-invariant closed subsets of $S^{n-1} \times S^{m-1}$.

Let $\hat{f}_{a}=f_{a} / \mathcal{G}$, i.e. $\hat{f}_{a}$, consists of equivalence class of the form $[(x, y)]=\{ \pm(x, y) \in$ $\left.f_{a}\right\} . \forall x \in f_{a}$, Let $j: S^{n-1} \rightarrow S^{m-1}$ be an odd $C^{1}$ map and $r>0$ be small. We set

$$
B=B(j, r):=\left\{(x, y) \in S^{n-1} \times S^{m-1} \mid y \in D_{r}(j(x))\right\},
$$

where $D_{r}\left(u_{0}\right)=\left\{u \in S^{m-1} \mid\left\|u-u_{0}\right\|<r\right\}$ is a disk.

It is easy to verify $B=B(j, r)$ is a $\mathcal{G}$ invariant set and is also a disk bundle: $D_{r} \rightarrow B \stackrel{\rho}{\longrightarrow} S^{n-1}$. 
Let $\hat{B}=B / \mathcal{G}$ be the quotient space consisting of equivalence classes $[(x, y)]=$ $\{ \pm(x, y) \in B\}$. Then $\hat{\rho}: \hat{B} \rightarrow \mathbb{R P}^{n-1}$ is a disk bundle with fibre $D_{r}$.

Definition 4.1. $A(p, q)$ order $(n, m)$ dimensional real tensor $\mathcal{A}$ is said to satisfy Condition (S) if there is an odd $C^{1}$ map

$$
j: S^{n-1} \rightarrow S^{m-1} \quad \text { and } \quad r>0 \text { small such that } B=B(j, r) \subset f_{0},
$$

where $f=f_{\mathcal{A}}$.

Lemma 4.2. If $\mathcal{A}$ satisfies Condition (S), then $\forall a>0$, $\operatorname{cat}_{\mathcal{G}}\left(\hat{f}_{a}\right) \geq \min (n, m)=n$.

Proof. First, we show $\operatorname{cat}_{\mathcal{G}}(\hat{B}(j, r))=n=\min (n, m)$. This is due to the facts:

$$
H^{*}\left(\hat{B} ; \mathbb{Z}_{2}\right) \cong H^{*}\left(\mathbb{R} \mathbb{P}^{n-1} \times D_{r} ; \mathbb{Z}_{2}\right) \cong H^{*}\left(\mathbb{R} \mathbb{P}^{n-1} ; \mathbb{Z}_{2}\right) \otimes H^{*}\left(D_{r} ; \mathbb{Z}_{2}\right) \cong H^{*}\left(\mathbb{R} \mathbb{P}^{n-1} ; \mathbb{Z}_{2}\right)
$$

and $\operatorname{cup}^{\mathrm{h}}\left(\mathbb{R P}^{n-1}\right)=n-1$.

Second, since $S^{n-1}$ is compact and both $f_{\mathcal{A}}$ and $j$ are continuous, assuming Condition (S), we have $r>0$ such that $B=B(j, r) \subset f_{a}$. It follows that

$$
n=\operatorname{cat}_{\mathcal{G}}(\hat{B}) \leq \operatorname{cat}_{\mathcal{G}}\left(\hat{f}_{a}\right) .
$$

We now have the following improvement:

Theorem 4.3. Assume that $\operatorname{cat}_{\mathcal{G}}(\hat{\Lambda})=s$ and that Condition (S) holds. If $p$ and $q$ are both odd, then, counting multiplicities, the function $f_{\mathcal{A}}(x, y)=\mathcal{A} x^{p} y^{q}$ has at least $r-s$ positive and $r-s$ negative critical values, where $r=\min \{n, m\}$.

Proof. Since $f_{\mathcal{A}}$ has only finitely many critical values, there exists $\epsilon>0$ such that $f_{\mathcal{A}}$ has no critical value in the interval $(0, \epsilon]$.

We apply Lemma 4.2 to $f_{\epsilon}$ and obtain: $\operatorname{cat}_{\mathcal{G}}\left(\hat{f}_{\epsilon}\right)=r$. We define critical values $c_{1} \leq c_{2} \leq \cdots \leq c_{r}$ as follows to see that

$$
c_{r}:=\inf _{\operatorname{cat}_{\mathcal{G}}(\hat{E}) \geq r} \max _{(x, y) \in \hat{E}} f_{\mathcal{A}}(x, y) \leq \max _{(x, y) \in f_{\epsilon}} f_{\mathcal{A}}(x, y) \leq \epsilon .
$$

By the choice of $\epsilon$, we have $c_{r} \leq 0$. We shall prove further that $c_{r-s}<0$.

If 0 is not a critical value of $f_{\mathcal{A}}$, then $c_{r-s} \leq c_{r}<0$. Otherwise, by letting $t=$ $\operatorname{cat}_{\mathcal{G}}\left(\hat{K}_{0}\right)$, where $K_{0}$ is the critical set with critical value 0 , we have $c_{r-t}<c_{r-t+1}=$ $\cdots=c_{r}=0$. By definition, $K_{0}=\Lambda$, and we assume $\operatorname{cat}_{\mathcal{G}}(\hat{\Lambda})=s$, this implies $t=s$. Thus, the existence of $r-s$ negative critical values (counting multiplicities) as well as the same number of positive critical values are established.

Corollary 4.4. Assume that $\operatorname{cat}_{\mathcal{G}}(\hat{\Lambda})=s$ and that Condition (S) holds. If $p$ and $q$ are both odd, then, counting multiplicities, the function $f_{\mathcal{A}}(x, y)=\mathcal{A} x^{p} y^{q}$ has at least $r-s$ eigenvectors $\left(x_{i}, y_{i}\right), i=1, \cdots, r-s$ with positive singular values and $r-s$ eigenvectors $\left(-x_{i}, y_{i}\right), i=1, \cdots, r-s$ with negative singular values, where $r=\min \{n, m\}$.

Corollary 4.5. Assume Condition (S) and $(p, q)$ both odd. If $\Lambda=\emptyset$ then, counting multiplicities, the function $f_{\mathcal{A}}$ has at least $r$ positive and $r$ negative critical values, where $r=\min \{n, m\}$.

It remains to answer when the Condition (S) holds for a tensor $\mathcal{A}$ with $f=f_{\mathcal{A}}$. We provide the following examples: 
EXAmPle 4.6. Let $\mathcal{A}$ be a $(p, 1)$ order $(n, m)$ dimensional tensor with $m \geq n$. Assume

$$
\mathcal{A} x^{p} \neq 0, \forall x \neq 0 \text {. }
$$

Then condition (S) holds.

We note:

$$
f_{0}=\left\{(x, y) \in S^{n-1} \times S^{m-1} \mid\left(\mathcal{A} x^{p}, y\right) \leq 0\right\}
$$

where $\mathcal{A} x^{p}$ is a nonzero vector in $\mathbb{R}^{m}$. We define:

$$
j(x)=-\frac{\mathcal{A} x^{p}}{\left\|\mathcal{A} x^{p}\right\|} \in S^{m-1} .
$$

Since

$$
f_{\mathcal{A}}(x, j(x))=-\left\|\mathcal{A} x^{p}\right\|<0, \forall x \in S^{n-1},
$$

and $S^{n-1}$ is compact, there is $r>0$ such that $f(x, y)<0 \forall y \in D_{r}(j(x))$, which implies (S).

EXAMPLE 4.7. If $\mathcal{A}$ is an $n \times m$ matrix, with rank $r \leq \min (n, m)$, then Condition $(S)$ holds.

In fact, if $r=\min (n, m)$, this is a special case of example 4.5. We now assume $r<\min (n, m)$. Let $R(\mathcal{A})$ be the range of $\mathcal{A}, R(\mathcal{A})^{\perp}$ be the orthogonal complement of $R(\mathcal{A})$ in $\mathbb{R}^{m}$, and $P$ be the orthogonal projection from $\mathbb{R}^{m}$ onto $R(\mathcal{A})^{\perp}$. Let $i: \mathbb{R}^{n} \rightarrow R^{m}$ be the inclusion.

Since $\operatorname{dim} R(\mathcal{A})^{\perp}=m-r, \operatorname{dim} R(i)=n$, and $n>r$, therefore $R(i) \bigcap R(\mathcal{A})^{\perp} \neq \emptyset$. The map

$$
j(x)=-\frac{P i(x)+\mathcal{A} x}{\|P i(x)+\mathcal{A} x\|}
$$

is well defined, and is a $C^{1}$ odd map.

From $\operatorname{Ker}\left(\mathcal{A}^{*}\right)=R(\mathcal{A})^{\perp}$, it follows that

$$
f_{\mathcal{A}}(x, j(x))=(\mathcal{A} x, j(x))=-\frac{\|\mathcal{A} x\|^{2}}{\|P i(x)+\mathcal{A} x\|} \leq 0,
$$

i.e., Condition (S) holds.

REMARK 4.8. According to Example 4.6, the matrix $\mathcal{A}$ possesses at least $r$ positive singular values and $-r$ negative singular values. This is an elementary fact in linear algebra. Thus, Theorem 4.3 serves as an extension of this fact to higher order tensors.

EXAmPle 4.9. Let $\mathcal{A}$ be $a(p, q)$ order $(n, m)$ dimensional tensor with $m \geq n$. Assume there is a linear subspace $Y \subset \mathbb{R}^{m}$ isomorphic to $\mathbb{R}^{n}$, i.e., $\exists i: \mathbb{R}^{n} \cong Y \subset \mathbb{R}^{m}$, such that $\mathcal{A} x^{p} i(x)^{q} \geq 0, \forall x \in S^{n-1}$; then Condition $(S)$ holds.

Reason: The isomorphism $-i$ induces an odd $C^{1}$ map $j: S^{n-1} \rightarrow S^{m-1}$ such that $\mathcal{A} x^{p} j(x)^{q} \leq 0, \forall x \in S^{n-1}$.

Acknowledgement. The authors would like to express their sincere gratitude to Prof. H. B. Duan for his helpful and valuable suggestions. 


\section{REFERENCES}

[1] A. Borel, Sur la cohomologie des espaces fibres princivaux et des espaces homogenes de groupes de Lie compactes, Ann. of Math., 57, 115-207, 1953.

[2] T. Bröcker and T.T. Dieck, Representations of Compact Lie Groups, GTM 98, Springer-Verlag, 1995.

[3] K.C. Chang, K. Pearson and T. Zhang, Perron-Frobenius theorem for nonnegative tensors, Commun. Math. Sci., 6, 507-520, 2008.

[4] K.C. Chang, K. Pearson and T. Zhang, On eigenvalue problems of real symmetric tensors, J. Math. Anal. Appl., 350, 416-422, 2009.

[5] K.C. Chang, L. Qi and G. Zhou, Singular Values of A Real Rectangular Tensor, preprint.

[6] O. Cornea, G. Lupton, J. Oprea and D. Tanre, Lusternik-Schnirelmann Category, Math. Surveys and Monographs 103, AMS, 2003.

[7] P. Comon, Tensor decompositions: state of the art and applications, in Math in Signal Processing, V (Coventry, UK, 2000), Insr. Math. Appl. Conf. Ser., Oxford Univ. Press, Oxford, UK, 71, 1-24, 2002.

[8] P. Comon, G. Golub, L.H. Lim and B. Mourrain, Symmetric tensors and symmetric tensor rank, SIAM J. Matrix Anal. Appl., 30(3), 1254-1279, 2008.

[9] M.W. Hirsch, Differential Topology, GTM 33 Springer, 1976.

[10] I.M. James, On category, in the sense of Lusternik-Schnirelmann, Topology, 17, 331-348, 1978.

[11] I.M. James, Lusternik-Schnirelmann Category, Handbook of Algebraic Topology, Ch. 27 Edited by I.M. James, 1293-1310, 1995.

[12] L. de Lathauwer, B. de Moor and J. Vandewalle, A multilinear singular value decomposition, SIAM J. Matrix Anal. Appl., 21(4), 1253-1278, 2000.

[13] L. de Lathauwer, B. de Moor and J. Vandewalle, On the best rank-1 and rank- $\left(R_{1}, \cdots, R_{N}\right)$ approximation of higher order tensors, SIAM J. Matrix Anal. Appl., 21(4), 1324-1342, 2000.

[14] L.H. Lim, Singular values and eigenvalues of tensors, a variational approach, Proc. 1st IEEE International Workshop on Computational Advances of Multi-tensor Adaptive Processing, 13-15, 129-132, 2005.

[15] W. Massey, On the cohomology ring of a sphere bundle, J. Math. Mech., 7, 265-289, 1958.

[16] J. Milnor and J. Stasheff, Characteristic Classes, Princeton University Press, 1974.

[17] M. Ng, L. Qi and G. Zhou, Finding the largest eigenvalue of a non-negative tensor, Manuscript, Department of Applied Mathematics, The Hong Kong Polytechnic University, January, 2009 .

[18] L. Qi, Eigenvalues of a real supersymmetric tensor, J. Symbolic Computation, 40, 1302-1324, 2005.

[19] L. Qi, Rank and eigenvalues of a supersymmetric tensor, a multivariate homogeneous polynomial and an algebraic surface defined by them, J. Symbolic Computation, 41, 1309-1327, 2006.

[20] L. Qi, Eigenvalues and invariants of tensors, J. Math. Anal. Appl., 325, 1363-1377, 2007.

[21] L. Qi, Y. Wang and E.X. Wu, D-eigenvalues of diffusion kurtosis tensor, J. Comput. Appl. Math., 221, 150-157, 2008.

[22] P.H. Rabinowitz, Minimax Methods in Critical Point Theory with Applications to Differential Equations, CBMS Regional Conference Series Math., AMS Porvidence, 65, 1986.

[23] Y.B. Rudyak, On the Ganea conjecture for manifolds, Proc. Amer. Math. Soc., 125(8), 25112512, 1997.

[24] E. Spanier, Algebraic Topology, McGraw-Hill, 1966.

[25] T. Zhang, Applications of algebraic topology in bounding the rank of the skew-symmetric curvature operator, Topology Appl., 124, 9-24, 2002. 